\title{
Evidence for Octupole Correlations in Multiple Chiral Doublet Bands
}

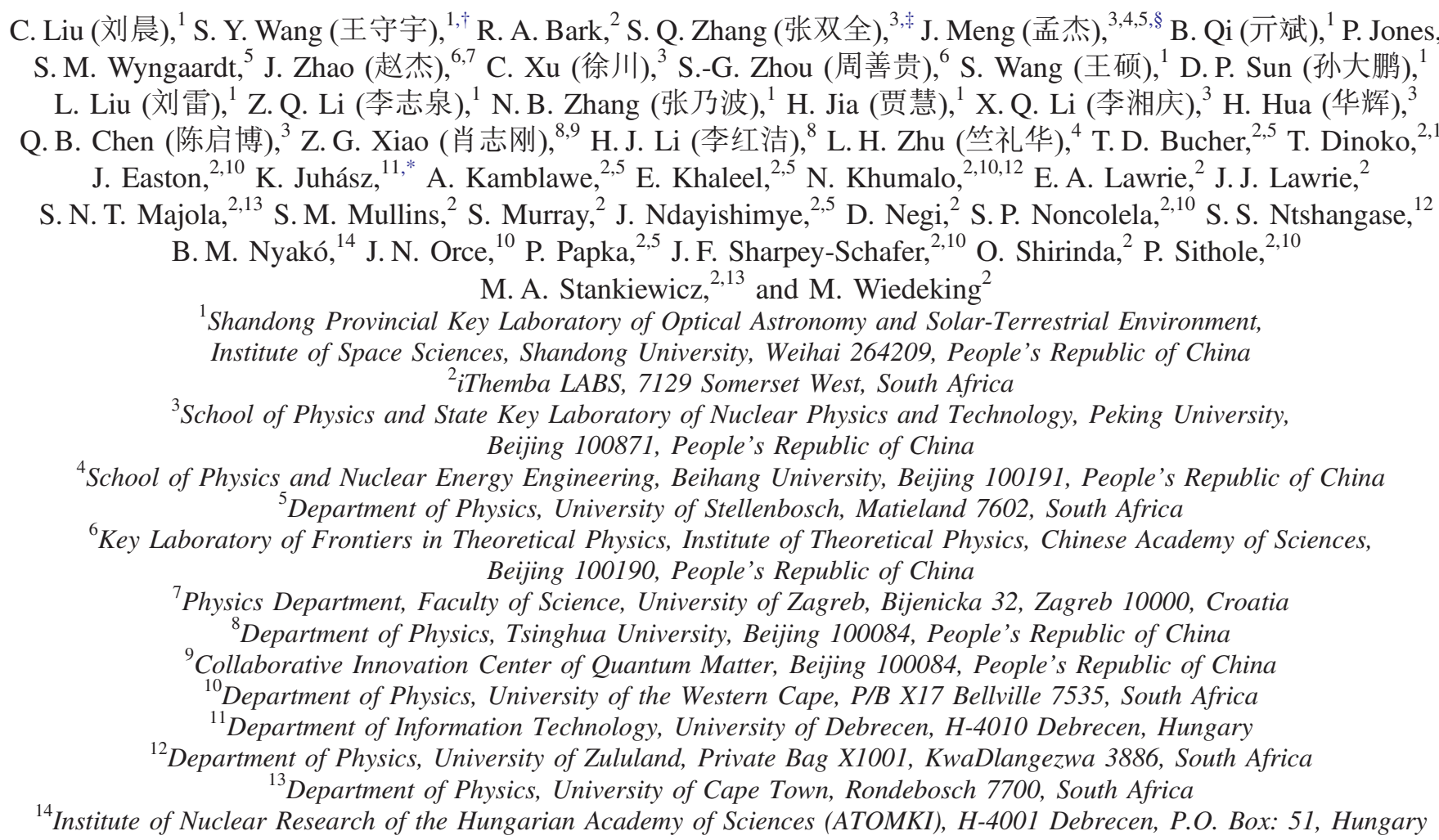

(Received 8 October 2015; revised manuscript received 2 December 2015; published 14 March 2016) transitions linking their yrast positive- and negative-parity bands have been identified in ${ }^{78} \mathrm{Br}$. They are interpreted as multiple chiral doublet bands with octupole correlations, which is supported by the microscopic multidimensionally-constrained covariant density functional theory and triaxial particle rotor model calculations. This observation reports the first example of chiral geometry in octupole soft nuclei.

DOI: 10.1103/PhysRevLett.116.112501

Spontaneous symmetry breaking is a fundamental concept in nature. As a many-body quantum system, the atomic nucleus carries a wealth of information on fundamental symmetries and symmetry breaking. As one example, chiral symmetry breaking in atomic nuclei has attracted considerable attention and intensive discussion since it was first predicted by Frauendorf and Meng [1]. They pointed out that, in the intrinsic frame of the rotating triaxial nucleus, the total angular momentum vector may lie outside the three principal planes, referred to as chiral geometry. The spontaneous chiral symmetry breaking in the laboratory frame may give rise to pairs of nearly degenerate $\Delta I=1$ bands with the same parity, i.e., chiral doublet bands. Such chiral doublet bands were first observed in $N=75$ isotones [2]. So far, more than 30 experimental candidates have been reported in the $A \sim 80,100,130$, and 190 mass regions [2-20].

Based on constrained triaxial covariant density functional theory (CDFT) calculations, it has been suggested that multiple chiral doublet $(\mathrm{M} \chi \mathrm{D})$ bands can exist in a single nucleus [21-26]. The theoretical prediction of $\mathrm{M} \chi \mathrm{D}$ bands stimulated lots of experimental efforts [27-31]. The first experimental evidence for $\mathrm{M} \chi \mathrm{D}$ bands was reported in ${ }^{133} \mathrm{Ce}$ [27], which confirmed the manifestation of triaxial shape coexistence in this nucleus. Later, Kuti et al. reported a novel type of $\mathrm{M} \chi \mathrm{D}$ bands with the same configuration in ${ }^{103} \mathrm{Rh}$ [29], which showed that chiral geometry can be robust against the increase of the intrinsic excitation energy.

Compared to the $A \sim 130$ and 100 mass regions, the $A \sim 80$ mass region is a relatively new and less studied territory for the investigation of chiral symmetry breaking in rotating nuclei, with only one report of chiral doublet bands based on the $\pi g_{9 / 2} \otimes \nu g_{9 / 2}$ configuration in odd-odd ${ }^{80} \mathrm{Br}$ [18]. In ${ }^{78} \mathrm{Br}$, the $\pi g_{9 / 2} \otimes \nu g_{9 / 2}$ band was suggested to have an obvious triaxial shape [32], which is suitable for the construction of chiral doublet bands. 
With $Z=35$ and $N=43$, valence protons and neutrons in ${ }^{78} \mathrm{Br}$ may occupy the orbits $g_{9 / 2}$ and $p_{3 / 2}$ with opposite parity and $\Delta j=\Delta l=3 \hbar$, which might lead to octupole correlations relating to the reflection symmetry breaking of the nuclear shape [33]. Indeed, experimental signals of octupole correlations in the 80 mass region have been reported [34-36].

Therefore, the investigation of $\gamma$-ray spectroscopy in ${ }^{78} \mathrm{Br}$ provides a unique opportunity to study chiral geometry, $\mathrm{M} \chi \mathrm{D}$ bands, and octupole correlations in a single nucleus. With both stable triaxial and octupole deformations, four $\Delta I=1$ rotational bands with the same configuration from the chiral and reflection symmetry breakings (chiralityparity quartet bands) are expected in one single nucleus. As the octupole deformation softens, two pairs of chiral doublets built on different single particle configurations with octupole correlations will appear.

In this Letter, we report on the first experimental evidence for $\mathrm{M} \chi \mathrm{D}$ bands with octupole correlations in ${ }^{78} \mathrm{Br}$.

The experiment was performed at iThemba LABS; medium- and high-spin states in ${ }^{78} \mathrm{Br}$ were populated using the reaction ${ }^{70} \mathrm{Zn}\left({ }^{12} \mathrm{C}, p 3 n\right)$ at beam energies of 60 and $65 \mathrm{MeV}$. The target was a self-supporting foil with a thickness of $0.85 \mathrm{mg} / \mathrm{cm}^{2}$. The deexciting $\gamma$ rays were recorded by the detector array AFRODITE [37], which comprised 8 Compton suppressed clover detectors. Four clovers were positioned at $135^{\circ}$ with respect to the beam direction, the remainder at $90^{\circ}$. To select specific chargeparticle reaction channels, the DIAMANT array $[38,39]$ of CsI particle detectors was also employed.

Approximately $1.5 \times 10^{9} \gamma-\gamma$ and $1.6 \times 10^{8} p-\gamma-\gamma$ coincidence events were collected. In the off-line analysis, the coincidence events were sorted into several symmetric and asymmetric matrices. The symmetric matrices were used for $\gamma-\gamma$ coincidence analyses, the asymmetric matrices gave angular distributions from the oriented states (ADO) [40] to obtain information on the multipolarities of $\gamma$ rays. For the present geometry, an ADO ratio $\sim 1.1$ is expected for the stretched quadrupole transitions and $\sim 0.7$ for the pure stretched dipoles. These values were obtained by extracting the ADO ratios of strong transitions in ${ }^{78} \mathrm{Kr}$ [41] whose multipolarities were already known. To distinguish the electric and magnetic character of the $\gamma$ rays, linear polarization measurements [42] were performed using two clover detectors positioned at $90^{\circ}$ relative to the beam direction as Compton polarimeters.

The level scheme of ${ }^{78} \mathrm{Br}$ obtained in the present work is shown in Fig. 1, with five bands labeled as 1-5. Prior to the present work, the ground state of ${ }^{78} \mathrm{Br}$ was assigned $I^{\pi}=1^{+}$with the $\pi p_{3 / 2} \otimes \nu p_{1 / 2}$ configuration $[43,44]$. Three isomeric states $\left(2^{-}\right.$at $32.3 \mathrm{keV}, 4^{+}$at $180.9 \mathrm{keV}$, and $5^{(+)}$at $227.7 \mathrm{keV}$ ) have been reported in Refs. [45-47].

Band 1
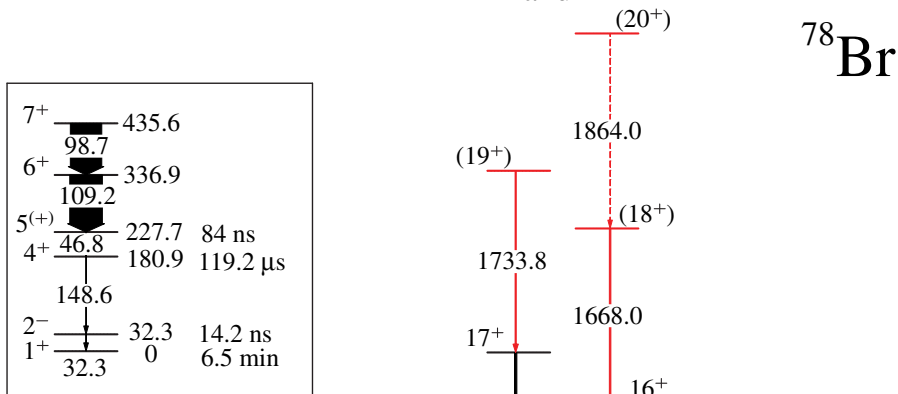

and 3

$\left(19^{-}\right.$

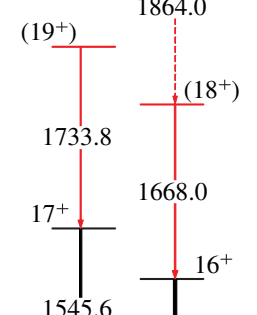

Band 5

$15^{(+)}$
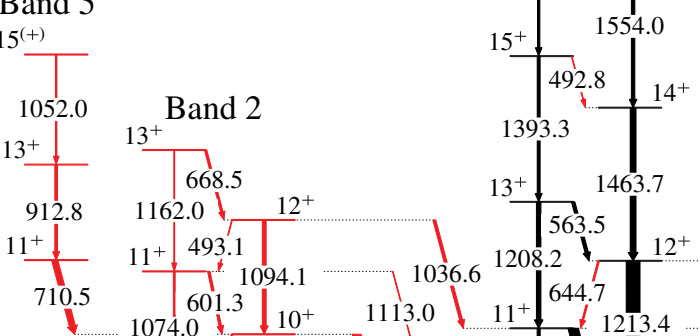

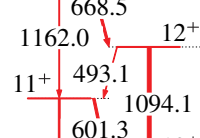

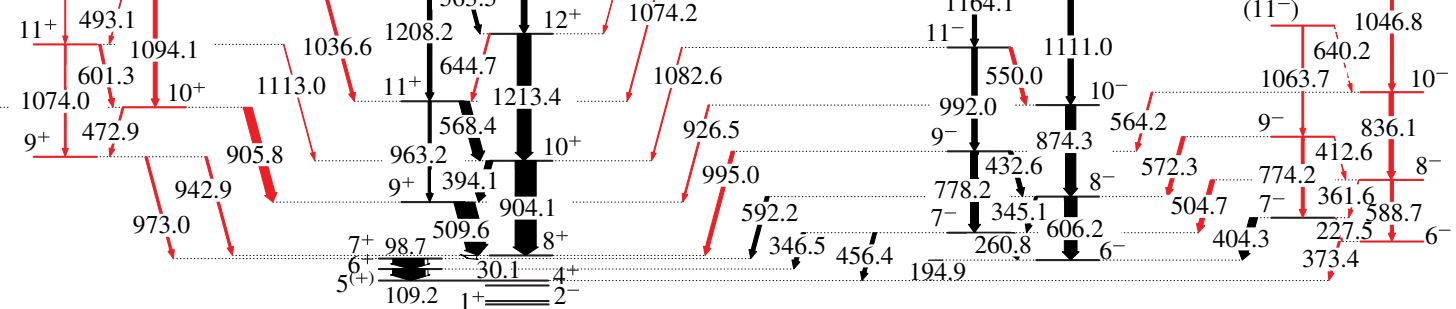

FIG. 1. Level scheme of ${ }^{78} \mathrm{Br}$ deduced from the present work. Transition energies are given in keV and their measured relative intensities are proportional to the widths of the arrows. New transitions and levels are marked as red. All the observed transitions in the present work eventually feed into the $5^{(+)}$state at $227.7 \mathrm{keV}$. The inset shows an expanded view of the lower part of the level scheme. 
The measured $g$ factors indicated that the $2^{-}$and $4^{+}$ isomeric states came from the $\pi p_{3 / 2} \otimes \nu g_{9 / 2}$ and $\pi g_{9 / 2} \otimes \nu g_{9 / 2}$ configurations, respectively [45,46]. It shows that the octupole orbitals $p_{3 / 2}$ and $g_{9 / 2}$ are active in ${ }^{78} \mathrm{Br}$. A positive-parity band (band 1 ) with the $\pi g_{9 / 2} \otimes \nu g_{9 / 2}$ configuration was first established in Ref. [47]. Landulfo et al. [32] further extended band 1 and observed another negative-parity band. However, no configuration assignment for the negative-parity band was given.

The present work extends the previously known level structures, and identifies three new bands labeled as 2, 4, and 5 as well as 15 new interband transitions. A total of 44 new transitions and 21 new levels are added to the level scheme. Figure 2 shows sample spectra supporting the present level scheme. The newly observed bands 2,4 , and 5 , respectively, feed into bands 1,3 , and 2 through several linking transitions. The measured $\mathrm{ADO}$ ratios $\left(R_{\mathrm{ADO}}\right)$ and the linear polarizations for the linking transitions in Fig. 3 suggest that the spin parity of the lowest observed state of bands 2,4 , and 5 are, respectively, $9^{+}, 6^{-}$, and $11^{+}$. The level depopulated by the $404.3 \mathrm{keV}$ interband transition from band 4 to the $6^{-}$state in band 3 was previously assigned as $I^{\pi}=\left(8^{-}\right)$[32]. However, as shown in Fig. 3, the extracted $\mathrm{ADO}$ value (0.79) and the linear polarization $(-0.07)$ indicate that the $404.3 \mathrm{keV}$ interband transition has an $M 1 / E 2$ character. We therefore propose the spin parity of this level is $7^{-}$instead of $\left(8^{-}\right)$.

One distinct feature of the level scheme is the presence of two pairs of nearly degenerate doublet bands (bands 1, 2 and 3,4). With the earlier configuration assignment $\pi g_{9 / 2} \otimes \nu g_{9 / 2}$ to band 1 [32] and the similar experimental features (energy spectra, energy staggering parameters, kinematic moment of inertia, and reduced transition probability ratios) to the chiral doublet bands observed in ${ }^{80} \mathrm{Br}$ [18], the positive-parity doublet bands 1 and 2 in ${ }^{78} \mathrm{Br}$ are therefore suggested as chiral doublet bands with the $\pi g_{9 / 2} \otimes \nu g_{9 / 2}$ configuration. The negative-parity bands 3

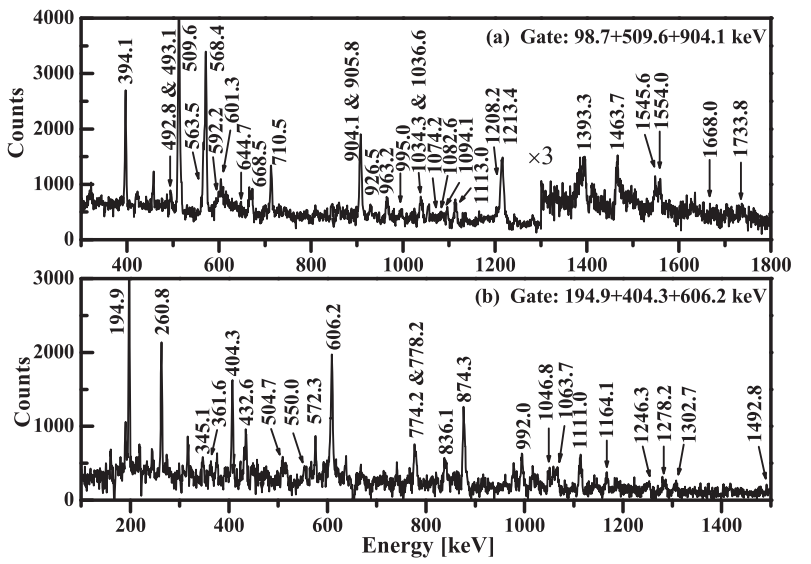

FIG. 2. The $\gamma$-ray coincidence spectra gated on the (a) $98.7+$ $509.6+904.1 \mathrm{keV}$ transitions and (b) $194.9+404.3+$ $606.2 \mathrm{keV}$ transitions in ${ }^{78} \mathrm{Br}$. and 4 form a pair of doublet bands with strong interconnecting transitions, suggesting the same intrinsic configurations for both bands. From the Nilsson diagram, the $\pi f_{5 / 2} \otimes \nu g_{9 / 2}$ and $\pi p_{3 / 2} \otimes \nu g_{9 / 2}$ configurations are candidates. Considering the configuration assignment $\pi p_{3 / 2} \otimes \nu g_{9 / 2}$ for the $2^{-}$isomer in ${ }^{78} \mathrm{Br}$ [45], we tentatively assign the $\pi f_{5 / 2} \otimes \nu g_{9 / 2}$ configuration to bands 3 and 4 .

With these configuration assignments for the two pairs of nearly degenerate bands, calculations based on a combination of the microscopic multidimensionally-constrained covariant density functional theory (MDC-CDFT) [48-50] and the triaxial particle rotor model (TPRM) [51-54] have been performed. The MDC-CDFT calculations with the PC-PK1 parameterization [55] show that the deformation parameters are $\left(\beta_{2}, \gamma\right)=\left(0.32,15.1^{\circ}\right)$ and $\left(0.23,26.3^{\circ}\right)$ for the $\pi g_{9 / 2} \otimes \nu g_{9 / 2}$ and $\pi f_{5 / 2} \otimes \nu g_{9 / 2}$ configurations, respectively. These deformation parameters are adopted in the TPRM calculations. The other parameters in TPRM follow Refs. [51-54,56]. The moments of inertia 16.0 and $18.0 \hbar^{2} / \mathrm{MeV}$ were used for bands 1,2 and bands 3, 4, respectively, while the Coriolis attenuation factor was set to 0.6 .

The excitation energies, energy staggering parameters $S(I)=[E(I)-E(I-1)] / 2 I$, and $B(M 1) / B(E 2)$ ratios calculated with TPRM for the positive-parity doublet bands 1 and 2 with the $\pi g_{9 / 2} \otimes \nu g_{9 / 2}$ configuration as well as the negative-parity doublet bands 3 and 4 with the $\pi f_{5 / 2} \otimes$ $\nu g_{9 / 2}$ configuration are shown in Fig. 4 , in comparison with the available data. The small energy differences between bands 1, 2 and bands 3, 4 are well reproduced, as well as the magnitude and trend of the energy staggering parameters

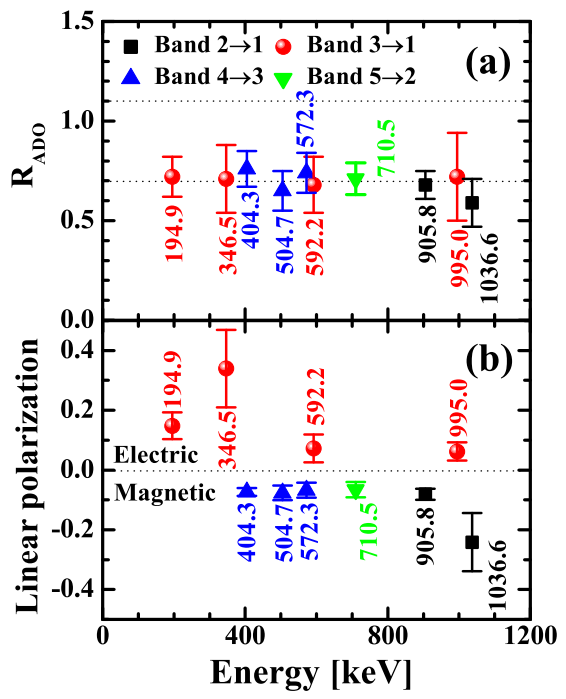

FIG. 3. The $R_{\mathrm{ADO}}$ (a) and linear polarizations (b) for the crucial linking transitions in ${ }^{78} \mathrm{Br}$. The dashed lines in the upper panel correspond to typical ADO values of stretched quadrupole transitions and stretched nonmixed dipole transitions. In the lower panel, a positive value corresponds to an electric transition and a negative value indicates a magnetic transition. 


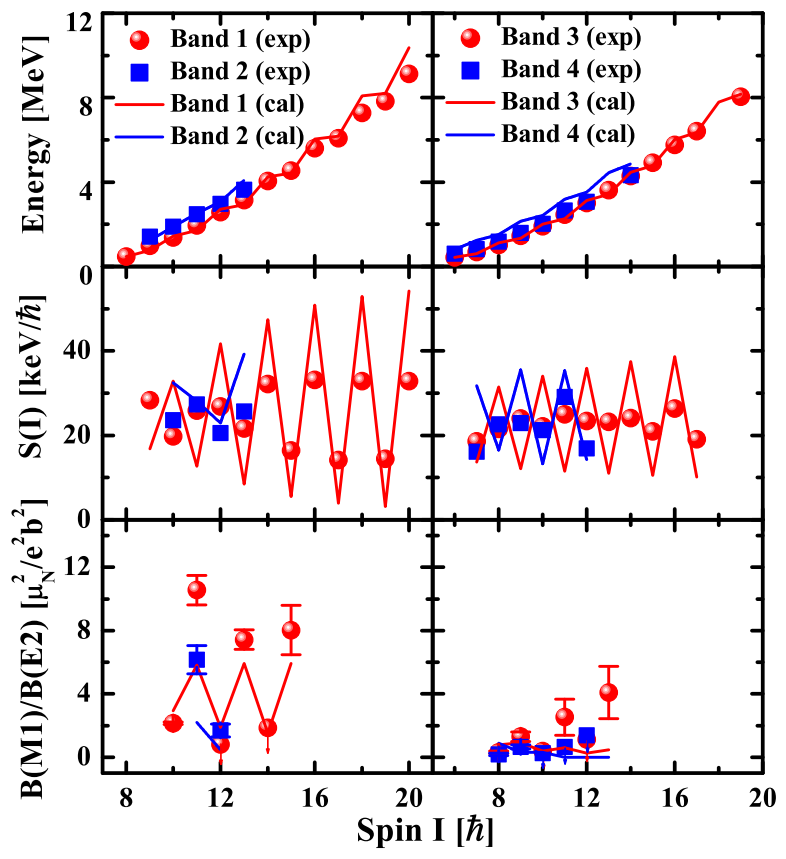

FIG. 4. The excitation energies, energy staggering parameters $S(I)=[E(I)-E(I-1)] / 2 I$, and $B(M 1) / B(E 2)$ ratios for the positive-parity chiral doublet bands 1,2 with the $\pi g_{9 / 2} \otimes \nu g_{9 / 2}$ configuration (left panels) and negative-parity chiral doublet bands 3,4 with the $\pi f_{5 / 2} \otimes \nu g_{9 / 2}$ configuration (right panels) in ${ }^{78} \mathrm{Br}$ in comparison with the TPRM results.

and $B(M 1) / B(E 2)$ ratios. In general, good agreement between the calculated values and the available experimental data are found, which supports the present configuration assignments. The deviation from the data for the $S(I)$ and $B(M 1) / B(E 2)$ ratios of band 3 might be attributed to the neglect of the mixing between the $f_{5 / 2}$ and $p_{3 / 2}$ orbitals in the TPRM calculations.

To investigate the chiral geometry in ${ }^{78} \mathrm{Br}$, the effective angles between the angular momentum vectors of the valence proton, valence neutron, and core for the two pairs of doublet bands are calculated in the TPRM following Refs. [53,57]. The effective angles are equal to or greater than $45^{\circ}$ for the two pairs of doublet bands in the observed spin region, which indicates clear nonplanar rotations for bands 1, 2 and bands 3, 4. This allows the two pairs of bands to be interpreted as pairs of chiral doublet bands, thereby forming $\mathrm{M} \chi \mathrm{D}$ bands.

One more distinct feature of the level scheme is the observation of eight $E 1$ linking transitions between the positive-parity band 1 and the negative-parity band 3 . The observation of the $E 1$ transitions between bands 1 and 3 implies the existence of the octupole correlations in ${ }^{78} \mathrm{Br}$. The configurations of bands 1 and 3 are currently interpreted as $\pi g_{9 / 2} \otimes \nu g_{9 / 2}$ and $\pi f_{5 / 2} \otimes \nu g_{9 / 2}$, respectively. The observation of the octupole correlations implies strong mixing between the $\pi p_{3 / 2}$ and $\pi f_{5 / 2}$ components due to triaxiality and pseudospin symmetry [58-61].
The interaction between the $\pi g_{9 / 2}$ and the $\pi p_{3 / 2}$ in the configurations leads to the octupole correlations in ${ }^{78} \mathrm{Br}$.

To study the octupole correlations in ${ }^{78} \mathrm{Br}$, the experimental $B(E 1) / B(E 2)$ branching ratios and the energy displacement $\delta E$ between the positive-parity band 1 and the negative-parity band 3 in ${ }^{78} \mathrm{Br}$ are extracted and compared with those in ${ }^{125} \mathrm{Ba}$ [62] and ${ }^{224} \mathrm{Th}$ [63] in Fig. 5. The energy displacement $\delta E(I)=E(I)-[(I+1) E(I-1)+$ $I E(I+1)] /(2 I+1)$ should be close to zero in the limit of stable octupole deformation $[36,64]$. The positive- and negative-parity bands connected by $E 1$ transitions in ${ }^{125} \mathrm{Ba}$ are based on different configurations and have been interpreted as octupole correlations [62]. The alternatingparity band in ${ }^{224} \mathrm{Th}$ was reported to have stable octupole deformation [63]. In Fig. 5(a), the $B(E 1) / B(E 2)$ branching ratios in ${ }^{78} \mathrm{Br}$ are comparable with those in ${ }^{125} \mathrm{Ba}$, but almost one order of magnitude smaller than ${ }^{224} \mathrm{Th}$. In Fig. 5(b), the energy displacements in ${ }^{78} \mathrm{Br}$ are also comparable with those in ${ }^{125} \mathrm{Ba}$, but deviate appreciably from those in ${ }^{224} \mathrm{Th}$. These results indicate that octupole correlations exist in ${ }^{78} \mathrm{Br}$. In Fig. 5, the $B(E 1) / B(E 2)$ branching ratios increase with spin in ${ }^{78} \mathrm{Br}$. The energy displacement stays constant for even spin and decreases dramatically for odd spin, which leads to the average decreasing trend. Both features indicate that the octupole correlations enhance with spin.

The octupole correlations in ${ }^{78} \mathrm{Br}$ are supported by the microscopic MDC-CDFT calculations as well. The potential energy surface in the $\beta_{20}-\beta_{30}$ plane for ${ }^{78} \mathrm{Br}$ calculated by the MDC-CDFT with the PC-PK1 parameterization is given in Fig. 6. The potential energy surface is very soft with respect to the shape degree of freedom $\beta_{30}$, which supports the octupole correlations in ${ }^{78} \mathrm{Br}$. Based on these

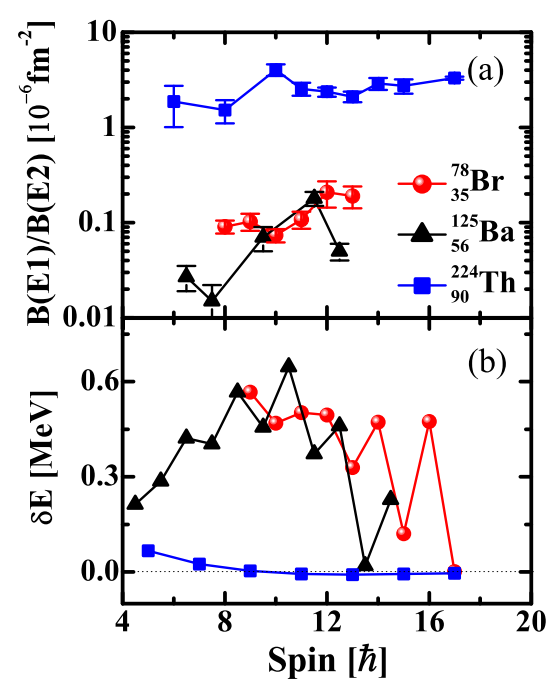

FIG. 5. The experimental $B(E 1) / B(E 2)$ ratios (a) and energy displacement $\delta E$ (b) between the positive- and negative-parity bands as a function of spin in ${ }^{78} \mathrm{Br}$, together with those in ${ }^{125} \mathrm{Ba}$ [62] and ${ }^{224} \mathrm{Th}[63]$. 


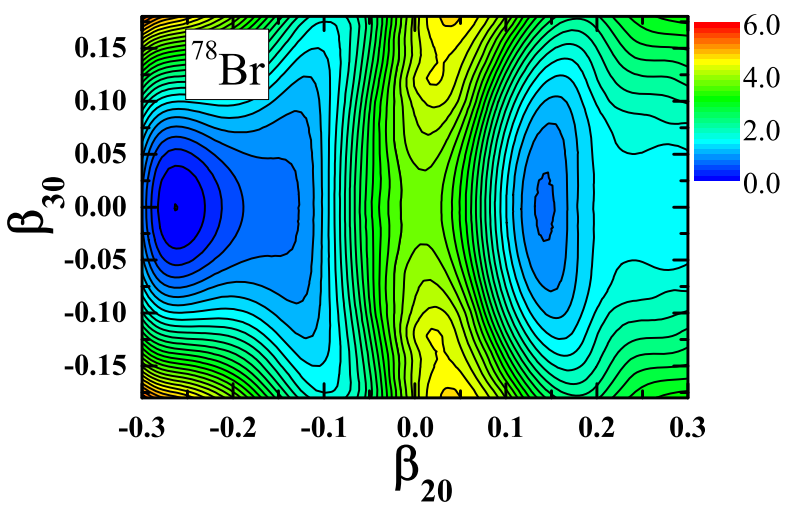

FIG. 6. The potential energy surface of ${ }^{78} \mathrm{Br}$ calculated using the MDC-CDFT approach. The energies are normalized with respect to the ground state. The contour separation is $0.2 \mathrm{MeV}$.

analyses, bands 1,2 and bands 3,4 in ${ }^{78} \mathrm{Br}$ can be interpreted as $\mathrm{M} \chi \mathrm{D}$ with octupole correlations. The observation of octupole correlations between the $\mathrm{M} \chi \mathrm{D}$ bands in ${ }^{78} \mathrm{Br}$ indicates that nuclear chirality can be robust against the octupole correlations and points to the exciting possibility of observing the chirality-parity quartet bands in a single nucleus.

In summary, two pairs of positive- and negative-parity doublet bands together with eight strong electric dipole transitions linking their yrast positive- and negative-parity bands, have been identified in ${ }^{78} \mathrm{Br}$. It provides the evidence for $\mathrm{M} \chi \mathrm{D}$ bands with octupole correlations, which is supported by the microscopic multidimensionallyconstrained covariant density functional theory and the triaxial particle-rotor model calculations. This observation reports the first example of chiral geometry in octupole soft nuclei and indicates that nuclear chirality can be robust against the octupole correlations. It is of highly scientific interest to search for the chirality-parity quartet bands in nucleus with both stable triaxial and octupole deformations.

Helpful discussions with S. Frauendorf and K. Starosta are gratefully acknowledged. This work is partly supported by the Natural Science Foundation of China under Grants No. 11175108, No. 11335002, No. 11175002, No. 11461141001, No. 11461141002, No. 11375015, No. U1432119, No. 11275248, No. 11525524 the Major State 973 Program of China (Grant No. 2013CB834400), the Research Fund for the Doctoral Program of Higher Education (Grant No. 20110001110087), and the SA/ CHINA research collaboration in science and technology under Grant No. CS05-L06. The authors thank the iThemba LABS technical staff and accelerator group for their support. Z. G.X. and H. J. L. are supported by the Tsinghua University Initiative Scientific Research Program. The computation of this work was partly supported by the HPC Cluster of SKLTP/ITP-CAS and the Supercomputing Center, CNIC of CAS.
*Deceased.

†sywang@sdu.edu.cn

*sqzhang@pku.edu.cn

\$mengj@pku.edu.cn

[1] S. Frauendorf and J. Meng, Nucl. Phys. A617, 131 (1997).

[2] K. Starosta et al., Phys. Rev. Lett. 86, 971 (2001).

[3] E. Grodner et al., Phys. Rev. Lett. 97, 172501 (2006).

[4] A. A. Hecht et al., Phys. Rev. C 63, 051302(R) (2001).

[5] D. J. Hartley et al., Phys. Rev. C 64, 031304(R) (2001).

[6] T. Koike, K. Starosta, C. J. Chiara, D. B. Fossan, and D. R. LaFosse, Phys. Rev. C 63, 061304(R) (2001).

[7] R. A. Bark, A. M. Baxter, A. P. Byrne, G. D. Dracoulis, T. Kibédi, T. R. McGoram, and S. M. Mullins, Nucl. Phys. A691, 577 (2001).

[8] T. Koike, K. Starosta, C. J. Chiara, D. B. Fossan, and D. R. LaFosse, Phys. Rev. C 67, 044319 (2003).

[9] S. Zhu et al., Phys. Rev. Lett. 91, 132501 (2003).

[10] J. Timár et al., Phys. Lett. B 598, 178 (2004).

[11] D. L. Balabanski et al., Phys. Rev. C 70, 044305 (2004).

[12] C. Vaman, D. B. Fossan, T. Koike, K. Starosta, I. Y. Lee, and A. O. Macchiavelli, Phys. Rev. Lett. 92, 032501 (2004).

[13] S. Y. Wang, Y.Z. Liu, T. Komatsubara, Y. J. Ma, and Y. H. Zhang, Phys. Rev. C 74, 017302 (2006).

[14] J. Timár, C. Vaman, K. Starosta, D. B. Fossan, T. Koike, D. Sohler, I. Y. Lee, and A. O. Macchiavelli, Phys. Rev. C 73, 011301(R) (2006).

[15] D. Tonev et al., Phys. Rev. Lett. 96, 052501 (2006).

[16] S. Mukhopadhyay et al., Phys. Rev. Lett. 99, 172501 (2007).

[17] E. A. Lawrie et al., Phys. Rev. C 78, 021305(R) (2008).

[18] S. Y. Wang et al., Phys. Lett. B 703, 40 (2011).

[19] E. Grodner et al., Phys. Lett. B 703, 46 (2011).

[20] P. L. Masiteng et al., Phys. Lett. B 719, 83 (2013).

[21] J. Meng, J. Peng, S. Q. Zhang, and S.-G. Zhou, Phys. Rev. C 73, 037303 (2006).

[22] J. Peng, H. Sagawa, S. Q. Zhang, J. M. Yao, Y. Zhang, and J. Meng, Phys. Rev. C 77, 024309 (2008).

[23] J. M. Yao, B. Qi, S. Q. Zhang, J. Peng, S. Y. Wang, and J. Meng, Phys. Rev. C 79, 067302 (2009).

[24] J. Meng and S. Q. Zhang, J. Phys. G 37, 064025 (2010).

[25] J. Li, S. Q. Zhang, and J. Meng, Phys. Rev. C 83, 037301 (2011).

[26] B. Qi, H. Jia, N. B. Zhang, C. Liu, and S. Y. Wang, Phys. Rev. C 88, 027302 (2013).

[27] A. D. Ayangeakaa et al., Phys. Rev. Lett. 110, 172504 (2013).

[28] D. Tonev et al., Phys. Rev. Lett. 112, 052501 (2014).

[29] I. Kuti et al., Phys. Rev. Lett. 113, 032501 (2014).

[30] E. O. Lieder et al., Phys. Rev. Lett. 112, 202502 (2014).

[31] N. Rather et al., Phys. Rev. Lett. 112, 202503 (2014).

[32] E. Landulfo et al., Phys. Rev. C 54, 626 (1996).

[33] P. A. Butler and W. Nazarewicz, Rev. Mod. Phys. 68, 349 (1996).

[34] R. B. Piercey, A. V. Ramayya, R. M. Ronningen, J.H. Hamilton, V. Maruhn-Rezwani, R. L. Robinson, and H. J. Kim, Phys. Rev. C 19, 1344 (1979).

[35] M. S. Kaplan, J.X. Saladin, L. Faro, D. F. Winchell, H. Takai, and C. N. Knott, Phys. Lett. B 215, 251 (1988).

[36] P. D. Cottle, Phys. Rev. C 41, 517 (1990). 
[37] R. A. Bark, M. Lipoglavsek, S. M. Maliage, S. S. Ntshangase, and A. Shevchenko, J. Phys. G 31, S1747 (2005).

[38] J. N. Scheurer et al., Nucl. Instrum. Methods Phys. Res., Sect. A 385, 501 (1997).

[39] J. Gal et al., Nucl. Instrum. Methods Phys. Res., Sect. A 516, 502 (2004).

[40] M. Piiparinen et al., Nucl. Phys. A605, 191 (1996).

[41] H. Sun et al., Phys. Rev. C 59, 655 (1999).

[42] P. M. Jones, L. Wei, F. A. Beck, P. A. Butler, T. Byrski, G. Duchene, G. de France, F. Hannachi, G. D. Jones, and B. Kharraja, Nucl. Instrum. Methods Phys. Res., Sect. A 362, 556 (1995).

[43] C. Ekstrom and L. Robertson, Phys. Scr. 22, 344 (1980).

[44] J. Prinz, I. Berkes, P. Herzog, B. Hlimi, M. DeJesus, M. Massaq, and I. Romanski, Hyperfine Interact. 75, 275 (1992).

[45] F. Pleiter, H. Bertschat, E. Recknagel, and B. Spellmeyer, Nucl. Phys. A215, 471 (1973).

[46] J. Christiansen, P. Heubes, H. Ingwersen, H. G. Johann, W. Klinger, W. Kreische, W. Lampert, W. Loeffler, G. Schatz, and W. Witthuhn, Rev. Roum. Phys. 17, 751 (1972).

[47] M. Behar, D. Abriola, A. Filevich, G. García Bermúdez, A. J. Kreiner, M. A. J. Mariscotti, J. A. Pinston, and D. Barneoud, Nucl. Phys. A376, 131 (1982).

[48] B. N. Lu, E. G. Zhao, and S.-G. Zhou, Phys. Rev. C 85, 011301(R) (2012).

[49] J. Zhao, B. N. Lu, E. G. Zhao, and S.-G. Zhou, Phys. Rev. C 86, 057304 (2012).
[50] B. N. Lu, J. Zhao, E. G. Zhao, and S.-G. Zhou, Phys. Rev. C 89, 014323 (2014).

[51] S. Y. Wang, S. Q. Zhang, B. Qi, and J. Meng, Phys. Rev. C 75, 024309 (2007).

[52] S. Q. Zhang, B. Qi, S. Y. Wang, and J. Meng, Phys. Rev. C 75, 044307 (2007).

[53] S. Y. Wang, S. Q. Zhang, B. Qi, J. Peng, J. M. Yao, and J. Meng, Phys. Rev. C 77, 034314 (2008).

[54] S. Y. Wang, B. Qi, and D. P. Sun, Phys. Rev. C 82, 027303 (2010).

[55] P. W. Zhao, Z. P. Li, J. M. Yao, and J. Meng, Phys. Rev. C 82, 054319 (2010).

[56] S. Y. Wang, B. Qi, and S. Q. Zhang, Chin. Phys. Lett. 26, 052102 (2009).

[57] B. Qi, S. Q. Zhang, J. Meng, S. Y. Wang, and S. Frauendorf, Phys. Lett. B 675, 175 (2009).

[58] A. Arima, M. Harvey, and K. Shimizu, Phys. Lett. B 30, 517 (1969).

[59] K. T. Hecht and A. Adler, Nucl. Phys. A137, 129 (1969).

[60] J. N. Ginocchio, Phys. Rep. 414, 165 (2005).

[61] H. Z. Liang, J. Meng, and S.-G. Zhou, Phys. Rep. 570, 1 (2015).

[62] P. Mason et al., Phys. Rev. C 72, 064315 (2005).

[63] B. Ackermann et al., Nucl. Phys. A559, 61 (1993).

[64] W. Nazarewicz and P. Olanders, Nucl. Phys. A441, 420 (1985). 